\title{
Rational HIV immunogen design to target specific germline B cell receptors
}

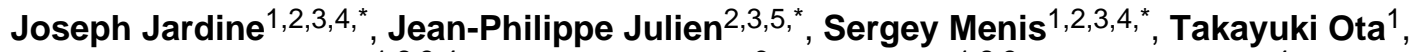 \\ Oleksandr Kalyuzhniy ${ }^{1,2,3,4}$, Andrew McGuire ${ }^{6}$, Devin Sok ${ }^{1,2,3}$, Po-Ssu Huang ${ }^{4}$, Skye \\ MacPherson ${ }^{1,2,3,4}$, Meaghan Jones ${ }^{1,2,4}$, Travis Nieusma ${ }^{2,3,5}$, John Mathison ${ }^{1}$, David Baker ${ }^{4}$, \\ Andrew B. Ward ${ }^{2,3,5}$, Dennis R. Burton ${ }^{1,2,3,7}$, Leonidas Stamatatos ${ }^{6,8}$, David Nemazee ${ }^{1}$, lan \\ A. Wilson $2,3,5,9$, and William R. Schief ${ }^{1,2,3,4,} \dagger^{\prime}$ \\ ${ }^{1}$ Department of Immunology and Microbial Science, The Scripps Research Institute, La Jolla, \\ California 92037, USA \\ ${ }^{2}$ IAVI Neutralizing Antibody Center, The Scripps Research Institute, La Jolla, California 92037, \\ USA \\ ${ }^{3}$ Center for HIV/AIDS Vaccine Immunology and Immunogen Discovery, The Scripps Research \\ Institute, La Jolla, California 92037, USA \\ ${ }^{4}$ Department of Biochemistry, University of Washington, Seattle, Washington, USA \\ ${ }^{5}$ Department of Integrative Structural and Computational Biology, The Scripps Research Institute, \\ La Jolla, California 92037, USA \\ ${ }^{6}$ Seattle Biomedical Research Institute, Seattle, WA 98109, USA \\ ${ }^{7}$ Ragon Institute of MGH, MIT, and Harvard, Cambridge, Massachusetts 02129, USA \\ ${ }^{8}$ Department of Global Health, University of Washington, Seattle, Washington, 98109 USA \\ ${ }^{9}$ Skaggs Institute for Chemical Biology, The Scripps Research Institute, La Jolla, California \\ 92037, USA
}

\section{Abstract}

\begin{abstract}
Vaccine development to induce broadly neutralizing antibodies (bNAbs) against HIV-1 is a global health priority. Potent VRC01-class bNAbs against the CD4 binding site of HIV gp120 have been isolated from HIV-1-infected individuals; however, such bNAbs have not been induced by vaccination. Wild-type gp120 proteins lack detectable affinity for predicted germline precursors of VRC01-class bNAbs, making them poor immunogens to prime a VRC01-class response. We employed computation-guided, in vitro screening to engineer a germline-targeting gp120 outer domain immunogen that binds to multiple VRC01-class bNAbs and their germline precursors. When multimerized on nanoparticles, this immunogen (eOD-GT6) activates both germline and mature VRC01-class B cells. Thus, eOD-GT6 nanoparticles have promise as a vaccine prime candidate. In principle, similar germline-targeting strategies can be applied to other epitopes and pathogens.
\end{abstract}

Protection against disease by nearly all licensed vaccines is associated with the induction of antibodies (1). Viruses with high antigenic diversity, such as HIV, Influenza, and hepatitis C virus, pose major challenges for vaccine development (2). Most exposed surfaces on the

\footnotetext{
†To whom correspondence should be addressed: schief@scripps.edu.

* These authors contributed equally to the work.
} 
Envelope glycoproteins (Env) of these viruses are hypervariable or shielded by glycans (3), and traditional vaccine approaches tend to induce neutralizing antibodies against only a small subset of viral strains (4-6). However, discoveries of bNAbs against each of these viruses have identified conserved epitopes as leads for vaccine design (2), and structural analysis has provided atomic definition for many of these epitopes $(7,8)$. Structure-based approaches are, therefore, needed to reverse-engineer vaccines capable of inducing bNAbs against these conserved epitopes (9).

High potency VRC01-class bNAbs against the HIV gp120 CD4 binding site (CD4bs) have been isolated from several rare individuals infected with different strains of HIV-1 (10-12). VRC01-class bNAbs all derive from the human VH1-2*02 variable heavy gene, but differ significantly in amino acid sequence and complementarity-determining region $\mathrm{H} 3$ (CDRH3) length and use a few different variable light chain genes (figs. S1-2). Structural studies have revealed that VRC01-class bNAbs employ a common mode of gp120 binding in which the VH1-2 framework mimics CD4 and provides additional electrostatic and hydrophobic contacts (Fig. 1A) (12-15). A short CDRL3 loop is also required for interaction with gp120 V5 and Loop D, and a CDRL1 deletion in many VRC01-class bNAbs avoids clashes with a glycan linked to gp120 Asn276 (N276). Vaccine design to induce VRC01-class bNAbs is attractive because VH1-2 genes are estimated to be present in $2 \%$ of the human $\mathrm{Ab}$ repertoire (16) and, even considering restrictions on light chain usage, suitable precursors should be present in the naïve B cell repertoire of most individuals. However, predicted germline (GL) precursors for VRC01-class bNAbs exhibit no detectable affinity for wildtype Env $(11,13)$ (Table 1 and table $S 1$ ), a potential explanation for the rarity of VRC01class bNAbs in HIV-1 infection (13). More importantly, wild-type Env constructs lacking GL affinity are poor vaccine candidates to prime VRC01-class responses, as they are unlikely to reliably stimulate GL precursors to initiate antibody maturation.

\section{Immunogen Design Strategy}

To address the problem described above, we modified the CD4bs on a minimal, "engineered outer domain (eOD)" (17) to produce a "germline-targeting" vaccine prime (Fig. 1) with two important binding properties: (i) moderate affinity for diverse predicted VH1-2*02 GL-Abs to enhance the ability to activate VH1-2 GL B cells with appropriate light chains; (ii) high affinity for VRC01-class bNAbs to provide an affinity gradient to guide early somatic mutation toward the mature bNAbs. Furthermore, we developed self-assembling nanoparticles presenting 60 copies of the germline-targeting eOD, to enhance B cell activation and to improve trafficking to lymph nodes (Fig. 1).

\section{Engineering and Biophysical Analysis of Germline-Targeting Antigens}

Modifications to the VRC01 epitope included removing clashes and building new contacts between the CD4bs and the GL-Abs, as well as rigidifying the CD4bs in a conformation that is favorable for binding. Initially, we constructed a homology model of GL-VRC01 bound to gp120 and identified a likely clash between CDRL1 and the N276 glycan. Therefore, we evaluated the GL-VRC01 binding of an eOD (eOD-Base) that lacks glycans at 276 and nearby on the V5 loop owing to N276D and N463D mutations. The eOD-Base barely interacted with GL-VRC01 $\left(K_{\mathrm{D}} \sim 1 \mathrm{mM}\right)$ and had low affinity for only 2 of 8 other GL VH1-2*02 Abs tested (Table 1). We then used Rosetta computational protein interface design (18) to identify other mutations in and around the CD4bs that were predicted to improve GL-VRC01 binding, and created directed libraries that included all possible combinations of the computationally identified mutations. These libraries were screened for core gp120 and eOD variants that showed GL-VRC01 binding using yeast cell surface display (19). This strategy generated germline-targeting (GT) variants of core gp120 
(CoreBal-GT1) and eOD (eOD-GT1) that bound GL-VRC01 with $K_{\mathrm{D}} \mathrm{s}$ of $1.8 \mu \mathrm{M}$ and 44 $\mu \mathrm{M}$, respectively (table S2). We focused further development on the smaller eOD because it lacks potentially distracting epitopes on core gp120. A second round of computational design and directed-library screening produced eOD-GT2 with a three-fold improvement in $K_{\mathrm{D}}$ for GL-VRC01 (table S2). Subsequent screening of mixed computational/random mutagenesis libraries led to larger improvements and resulted in eOD-GT3 and eOD-GT4, which had $K_{\mathrm{D}} \mathrm{s}$ for GL-VRC01 of 220 and $34 \mathrm{nM}$, respectively (table S2). Interestingly, screening for GL-VRC01 binding also improved binding to other GL VH1-2 Abs, as eODGT4 bound to GL-NIH45-46 and GL-PGV19 with $K_{\mathrm{D}} \mathrm{s}$ of $1.0 \mu \mathrm{M}$ and $28 \mathrm{nM}$, respectively (table S2). To achieve these improvements, eOD-GT4 had accumulated 17 mutations relative to eOD-Base.

To retain as native a CD4bs as possible (by reducing the number of mutations) while also maintaining or improving binding to GL VH1-2 Abs and mature bNAbs, we employed multi-target optimization. Here, libraries with either the wild-type HIV-1 strain HxB2 residue or the mutation in eOD-GT4 were sorted in parallel against multiple Abs, and mutations were retained only if they were beneficial to at least one GL Ab and tolerated by all Abs in the sort. eOD-GT6 was generated by sorting against GL Abs for VRC01, NIH45-46, PGV19, PGV04 and VRC-CH31, as well as mature VRC01 and PGV04. eODGT6 had only 8 mutations relative to eOD-base (10 mutations relative to HxB2 gp120) and retained excellent binding to diverse GL VH1-2*02 Abs, with a $K_{\mathrm{D}}$ of $44 \mathrm{nM}$ for GLVRC01 and $K_{\mathrm{D}} \mathrm{s}<500 \mathrm{nM}$ for 5 of 9 GL Abs tested (Table 1, table S2, fig. S3). eOD-GT6 also had high affinity for several mature bNAbs, with $K_{\mathrm{D}}^{\mathrm{S}}$ of 2, 4, and $88 \mathrm{nM}$ for VRC01, NIH45-46, and PGV19, respectively. eOD-GT6 also maintained the desired affinity gradient for 6 of 8 Abs tested (Table 1). Further, eOD-GT6 bound with high affinity to GL VRC01class Abs derived from VH1-2*03 and *04 alleles (table S3, fig. S4). eOD-GT6 had no affinity for $\mathrm{VH} 1-2 * 01$, probably due to the absence of $\operatorname{Trp}^{\mathrm{H} 50}$, however, recent data from sequencing of 1092 human genomes(20)shows that the W50R mutation in VH1-2*01occurs at a frequency of 0.21 , indicating that only $\sim 4 \%$ of the population are $\mathrm{VH} 1-2 * 01$ homozygotes not amenable to eOD-GT6 priming.

\section{Crystallographic Analysis}

To understand the atomic interaction of eOD-GT6 with GL-VRC01, we solved three crystal structures: unliganded GL-VRC01, unliganded eOD-GT6, and the complex of GL-VRC01 bound to eOD-GT6, to resolutions of $2.1 \AA$, $2.5 \AA$, and $2.4 \AA$, respectively (Fig 2 and table S4). The structure of unliganded GL-VRC01 revealed that the gp120 contacting loops closely resemble those of VRC01 despite extensive affinity maturation of the latter (Fig. 2A, and figs. S5-7). Unliganded eOD-GT6 showed a similar structure to the outer domains of unliganded and VRC01-bound gp120 core (1.2 ̊ Ca root-mean-square-deviation (RMSD) in both instances) (Figs. 2B and fig. S8), suggesting good mimicry of the CD4bs. The eODGT6 unliganded structure was also similar to the structure of the eOD-GT6 bound to GLVRC01 Fab (0.9 Å RMSD), with the largest differences occurring in the flexible loops and in the eOD exit loop (fig. S9). In addition, the crystal structure of the eOD-GT6+GL-VRC01 complex indicated that the VH1-2-encoded domain of GL-VRC01 has an angle of approach to eOD-GT6 nearly identical to that of VRC01 to gp120 (Fig. 2C) $\left(4.2^{\circ}\right.$ angular difference when the complexes are superposed on the CD4b loop). Overall, the buried surface area (BSA) of GL-VRC01 $\left(1075.6 \AA^{2}\right)$ on eOD-GT6 $\left(1102.0 \AA^{2}\right)$ is nearly identical to VRC01 $\left(1125.7 \AA^{2}\right)$ on core gp120 (1158.2 $\left.\AA^{2}\right)$, further demonstrating the high degree of similarity between the two structures (tables S5-6). Key hydrogen-bonding networks are preserved in the GL-VRC01+eOD-GT6 interaction, particularly in the CD4b loop (fig. S10). On the other hand, key differences in hydrogen bonds, BSA and $\mathrm{Ca}$ positions are observed for 
interactions in loop D, V5 and the OD exit loop, which we hypothesize confer GL-VRC01 reactivity to eOD-GT6 (Fig. 2D and fig. S11).

\section{Reversion Analysis}

To understand the affinity contributions of individual mutations, we measured GL-VRC01 binding affinities for point reversions of each mutation (Table 2). Six mutations on eODGT6 conferred improved affinity for GL-Abs relative to the starting construct (eOD-Base) that lacked glycans at 276 and 463 . The eOD-GT6+GL-VRC01 complex crystal structure revealed that these mutations are either directly involved in the binding interface (T278R, I371F and N460V) or stabilize loops involved in the interface (L260F, K357R and G471S) (Fig. 2C). The two mutations with the largest effect on GL-VRC01 binding were G471S and I371F; reversion at these positions reduced GL-VRC01 affinity by factors of 39 and 10, respectively (Table 2). Ser471, together with Phe371 and Phe260 appears to play a role in altering the conformation of the OD exit loop to allow the GL-VRC01 CDRH2 to make Hbonds with three additional gp120 residues (G472, G473 and D474) and bury an additional $119.6 \AA^{2}$ on gp120, resulting in improved binding (Fig. 2C-D, inset 2, tables S5-6, fig. S9). Also at the interface, the N460V mutation located in V5 improves packing with the antibody and appears to contribute to an altered V5 conformation and pattern of V5 H-bonding with VRC01, as compared to Clade A/E 93TH057 gp120 recognition of VRC01 (Fig. 2D and fig. S12). Reversion of the N460V mutation reduced GL-VRC01 binding by a factor of 2.5 (Table 2).

Removal of key glycosylation sites was necessary for GL affinity. Reintroduction of the N276 glycosylation site in eOD-GT6 (by a double mutation, D276N/R278T) reduced binding by a factor of 140 , and the remaining binding was likely due to a small fraction of the eOD-GT6-D276N/R278T that underutilized the N276 glycosylation position (fig. S13). Reversion of R278T alone reduced affinity by a factor of only 3.6 (Table 2). Thus, removal of the 276 glycan appears to release a block on GL-VRC01 binding but does not confer appreciable eOD affinity; further interface modification was required to achieve high affinity. Indeed, the eOD-GT6+GL-VRC01 complex crystal structure revealed that, in addition to removing a clash between the N276 glycan on gp120 and the GL-VRC01 CDRL1 (Fig. 2C, inset 1), eOD-GT6 residues D276 and R278 make two additional H-bonds with GL-VRC01. eOD-GT6 also lacks glycans at positions 386 ( $\beta 12)$ and 463 (V5). Restoration of these glycosylation sites reduced affinity for GL-VRC01 by a factor of 3 (table S7).

\section{eOD-GT6 Nanoparticle Generation}

To enable eOD-GT6 to activate GL B cells via cross-linking of B cell receptors, and to develop a multivalent platform for eOD-GT6 that mimics the size, shape, and symmetric surface geometry of many viruses for improved immunogenicity (21), we sought to fuse eOD-GT6 to a self-assembling virus-like nanoparticle. From a search of large homomeric particles in the Protein Data Bank (PDB) we prioritized 60mers of Lumazine Synthase from the hyperthermophile Aquifex Aeolicus for experimental testing, due to their thermal stability and because modeling suggested that, with a suitable linker length, 60 copies of glycosylated eOD-GT6 could be sterically accommodated in an orientation that would expose the VRC01 epitope (Fig 3A). Though expression of the wild-type particle had been reported in E. coli (22), we found that such nanoparticles presenting glycosylated eOD-GT6 could be secreted from mammalian (293) cells and purified by lectin chromatography with good yield ( 10 mg/L) and structural homogeneity (Fig 3B and figs S14-15). 


\section{In Vitro B Cell Activation}

The ability of eOD-GT6 nanoparticles to activate B-cells expressing GL and mature VRC01 (IgM) (23), 12A12 (IgM) and NIH45-46 (IgG) (24), was tested in $\mathrm{Ca}^{2+}$-dependent activation assays. The 60 -mers potently activated both GL and mature B cells with $1 \mu \mathrm{M}$ outer domain $(16 \mathrm{nM}$ particle) and modestly activated all three cell lines at 1000-fold lower concentrations (Fig. 3C and fig. S16). In contrast, monomeric eOD-GT6 was non-stimulatory, probably due to an inability to cross-link B cell receptors (23). Trimeric eOD-GT6 activated both GL and mature B cells but less potently and rapidly than the 60mers, and a soluble gp 140 trimer from HIV-1 strain YU2 (25) showed no activation of GL B cells but did activate the mature counterparts (Fig. 3C). Both IgM and IgG B cell lines were generated for GL 12A12 and we observed no significant difference between activation behaviors of the two antibody isotypes (fig. S16).

\section{Animal Models for Human VH1-2 Germline-Targeting}

We then assessed whether eOD-GT6 might interact with related GL-Abs in animal models. Analysis of VH genes from rabbit (fig. S17) (26), mouse (figs. S18-19) (27) and macaque (fig. S20) revealed that none of these commonly used model organisms have a known VH gene containing all of the critical residues for GL binding (15). To measure binding experimentally, chimeric GL-Abs were produced in which the human VH1-2*02 gene from GL-VRC01 was replaced with GL VH genes from mice or macaques containing the essential $\mathrm{Arg}^{\mathrm{H} 71}$ and as many other critical residues as possible. Chimeric GL-Abs with mouse $\mathrm{VH}$ genes had no detectable binding to eOD-GT6. Abs derived from two of the three rhesus VH genes bound only weakly to eOD-GT6, with $K_{\mathrm{D}}$ s of $\sim 30 \mu \mathrm{M}$ and $\sim 40 \mu \mathrm{M}$ (table S8). Despite being $>94 \%$ identical in sequence to human GL-VRC01, the rhesus chimeric GL-Abs had three orders of magnitude lower affinity. Annotation of the rhesus macaque antibody repertoire and analysis of gene usage frequencies will be useful to construct bona fide macaque GL VH1-2 Abs. These analyses illustrate the potential difficulty for using animal models to produce VRC01-class bNAbs, and suggest that immunization of humans or mice engineered to produce human Abs may be essential for testing and iteratively optimizing such immunogens.

The events that led to GL VH1-2*02 B cell activation in the HIV-infected individuals from which VRC01-class bNAbs were isolated remain unclear. Our finding that a small number of rare or previously undocumented Env mutations confers high affinity GL binding suggests that Env variants might have acquired one or more such mutations stochastically during infection and thereby gained the ability to prime GL VRC01-class B cells. Vaccines to induce VRC01-class responses will need to activate such B cells dependably and drive appropriate somatic mutation to produce high affinity bNAbs (28). We propose the eODGT6 nanoparticle as a promising candidate for a vaccine prime based on its ability to bind diverse VH1-2*02 GL Abs, activate VRC01, 12A12 and NIH45-46 B cells in vitro, and provide an affinity gradient for early somatic mutation. We hypothesize that ultimate elicitation of mature VRC01-class bNAbs will require, at minimum, boosting with different immunogens that present a less engineered, more native CD4bs including the glycans around the CD4bs.

\section{Supplementary Material}

Refer to Web version on PubMed Central for supplementary material. 


\section{Acknowledgments}

We thank J. Mascola and X. Wu for providing sequences of mature PGV19 and PGV20, R. Wyatt for providing the YU2 gp140 trimer, C. Sundling and G. Karlsson Hedestam for the sequence of Rhe1 VH gene, and A. Bradley, R.L. Stanfield, and D.C. Diwanji for technical assistance and insightful discussions. The data presented in this manuscript are tabulated in the main paper and the supplementary materials. Coordinates and structure factors for GL-VRC01 Fab, eOD-GT6 and GL-VRC01+eODGT6 structures will be deposited with the Protein Data Bank and released upon publication. The International AIDS Vaccine Initiative (IAVI) has filed a patent relating to immunogens in this manuscript: PCT Application No. PCT/US12/60062, titled "Engineered Outer Domain (EOD) Of HIV GP120 And Mutants Thereof", with inventors WRS, JJ, SM, PH. Materials and information will be provided under MTA. This work was supported by the International AIDS Vaccine Initiative Neutralizing Antibody Center, CHAVI-ID (UM1 AI100663), NIH grant AI84817 (I.A.W.), National Institute of Allergy and Infectious Diseases grants P01AI081625 (L.S.) and AI33292 (D.R.B.), NIH NRSA Training Grant fellowship T32CA080416 (J.J.), Canadian Institutes of Health Research fellowship (J.-P.J.), CNIHR grant P30 AI027767-24 (T.O.), NIH Interdisciplinary Training Program in Immunology 5T32AI007606-10 (D.S.) and the Ragon Institute. Portions of this research were carried out at the Stanford Synchrotron Radiation Lightsource (SSRL), a Directorate of the SLAC National Accelerator Laboratory and an Office of Science User Facility operated for the U.S. Department of Energy (DOE) Office of Science by Stanford University. The SSRL Structural Molecular Biology Program is supported by the DOE Office of Biological and Environmental Research; NIH's National Center for Research Resources, Biomedical Technology Program (P41RR001209); and the National Institute of General Medical Sciences (NIGMS). Use of the Advanced Photon Source was supported by the DOE, Basic Energy Sciences, Office of Science, under contract no. DE-AC02-06CH11357. GM/CA CAT has been funded in whole or in part with federal funds from NCI (grant Y1-CO-1020) and NIGMS (grant Y1-GM-1104). This is manuscript 23050 from The Scripps Research Institute.

\section{References}

1. Plotkin SA. Correlates of protection induced by vaccination. Clin Vaccine Immunol. 2010; 17:1055. [PubMed: 20463105]

2. Burton DR, Poignard P, Stanfield RL, Wilson IA. Broadly neutralizing antibodies present new prospects to counter highly antigenically diverse viruses. Science. 2012; 337:183. [PubMed: 22798606]

3. Schief WR, Ban YEA, Stamatatos L. Challenges for structure-based HIV vaccine design. Curr Opin HIV AIDS. 2009; 4:431. [PubMed: 20048708]

4. Burton DR, et al. A Blueprint for HIV Vaccine Discovery. Cell Host Microbe. 2012; 12:396. [PubMed: 23084910]

5. Ekiert DC, et al. Antibody recognition of a highly conserved influenza virus epitope. Science. 2009; 324:246. [PubMed: 19251591]

6. Zhang $\mathrm{P}$, et al. Depletion of interfering antibodies in chronic hepatitis $\mathrm{C}$ patients and vaccinated chimpanzees reveals broad cross-genotype neutralizing activity. Proc Natl Acad Sci U S A. 2009; 106:7537. [PubMed: 19380744]

7. Julien JP, Lee PS, Wilson IA. Structural insights into key sites of vulnerability on HIV-1 Env and influenza HA. Immunol Rev. 2012; 250:180. [PubMed: 23046130]

8. Kong L, et al. Structural basis of hepatitis $\mathrm{C}$ virus neutralization by broadly neutralizing antibody HCV1. Proc Natl Acad Sci U S A. 2012; 109:9499. [PubMed: 22623528]

9. Burton DR. Antibodies, viruses and vaccines. Nat Rev Immunol. 2002; 2:706. [PubMed: 12209139]

10. Wu XL, et al. Rational design of envelope identifies broadly neutralizing human monoclonal antibodies to HIV-1. Science. 2010; 329:856. [PubMed: 20616233]

11. Scheid JF, et al. Sequence and structural convergence of broad and potent HIV antibodies that mimic CD4 binding. Science. 2011; 333:1633. [PubMed: 21764753]

12. Wu X, et al. Focused evolution of HIV-1 neutralizing antibodies revealed by structures and deep sequencing. Science. 2011; 333:1593. [PubMed: 21835983]

13. Zhou TQ, et al. Structural basis for broad and potent neutralization of HIV-1 by antibody VRC01. Science. 2010; 329:811. [PubMed: 20616231]

14. Diskin R, et al. Increasing the potency and breadth of an HIV antibody by using structure-based rational design. Science. 2011; 334:1289. [PubMed: 22033520] 
15. West AP Jr, Diskin R, Nussenzweig MC, Bjorkman PJ. Structural basis for germ-line gene usage of a potent class of antibodies targeting the CD4-binding site of HIV-1 gp120. Proc Natl Acad Sci U S A. 2012; 109:E2083. [PubMed: 22745174]

16. Arnaout R, et al. High-resolution description of antibody heavy-chain repertoires in humans. Plos One. $2011 ; 6$

17. Pejchal R, et al. A potent and broad neutralizing antibody recognizes and penetrates the HIV glycan shield. Science. 2011; 334:1097. [PubMed: 21998254]

18. Leaver-Fay A, et al. ROSETTA3: an object-oriented software suite for the simulation and design of macromolecules. Methods Enzymol. 2011; 487:545. [PubMed: 21187238]

19. Chao G, et al. Isolating and engineering human antibodies using yeast surface display. Nature Protocols. 2006; 1:755.

20. C. Genomes Project et al. An integrated map of genetic variation from 1,092 human genomes. Nature. 2012; 491:56. [PubMed: 23128226]

21. Bachmann MF, Jennings GT. Vaccine delivery: a matter of size, geometry, kinetics and molecular patterns. Nat Rev Immunol. 2010; 10:787. [PubMed: 20948547]

22. Zhang X, Meining W, Fischer M, Bacher A, Ladenstein R. X-ray structure analysis and crystallographic refinement of lumazine synthase from the hyperthermophile Aquifex aeolicus at 1.6 Å resolution: determinants of thermostability revealed from structural comparisons. J Mol Biol. 2001; 306:1099. [PubMed: 11237620]

23. Ota T, et al. Anti-HIV B cell lines as candidate vaccine biosensors. J Immunol. 2012

24. Hoot S, et al. Recombinant HIV Envelope Proteins Fail to Engage Germline Versions of AntiCD4bs bNAbs. PLoS Pathog. 2013; 9:e1003106. [PubMed: 23300456]

25. Sundling C, et al. Soluble HIV-1 Env trimers in adjuvant elicit potent and diverse functional B cell responses in primates. J Exp Med. 2010; 207:2003. [PubMed: 20679401]

26. Knight KL, Winstead CR. Generation of antibody diversity in rabbits. Curr Opin Immunol. 1997; 9:228. [PubMed: 9099798]

27. Several mouse VH genes were identified that contained the ArgH71 important for eOD-GT6 binding, but crystal structures of mouse Abs indicated that ArgH71 would not be accessible for binding, as it forms internal H-bonds with backbone carbonyl groups (fig. S15).

28. Haynes BF, Kelsoe G, Harrison SC, Kepler TB. B-cell-lineage immunogen design in vaccine development with HIV-1 as a case study. Nat Biotechnol. 2012; 30:423. [PubMed: 22565972]

29. Krissinel E, Henrick K. Inference of macromolecular assemblies from crystalline state. J Mol Biol. 2007; 372:774. [PubMed: 17681537]

30. Pettersen EF, et al. UCSF Chimera--a visualization system for exploratory research and analysis. J Comput Chem. 2004; 25:1605. [PubMed: 15264254]

31. Souto-Carneiro MM, Longo NS, Russ DE, Sun HW, Lipsky PE. Characterization of the human Ig heavy chain antigen binding complementarity determining region 3 using a newly developed software algorithm, JOINSOLVER. J Immunol. 2004; 172:6790. [PubMed: 15153497]

32. Huang P-S, et al. RosettaRemodel: a generalized framework for flexible backbone protein design. Plos One. 2011; 6

33. Kuhlman B, et al. Design of a novel globular protein fold with atomic-level accuracy. Science. 2003; 302:1364. [PubMed: 14631033]

34. Fleishman SJ, et al. RosettaScripts: a scripting language interface to the rosetta macromolecular modeling suite. Plos One. 2011; 6

35. Gray JJ, et al. Protein-protein docking with simultaneous optimization of rigid-body displacement and side-chain conformations. J MolBiol. 2003; 331:281.

36. Davis IW, Arendall WB, Richardson DC, Richardson JS. The backrub motion: How protein backbone shrugs when a sidechain dances. Structure. 2006; 14:265. [PubMed: 16472746]

37. Stemmer WP, Crameri A, Ha KD, Brennan TM, Heyneker HL. Single-step assembly of a gene and entire plasmid from large numbers of oligodeoxyribonucleotides. Gene. 1995; 164:49. [PubMed: 7590320] 
38. Benatuil L, Perez JM, Belk J, Hsieh CM. An improved yeast transformation method for the generation of very large human antibody libraries. Protein Eng Des Sel. 2010; 23:155. [PubMed: 20130105]

39. Bryson S, et al. Cross-neutralizing human monoclonal anti-HIV-1 antibody 2F5: Preparation and crystallographic analysis of the free and epitope-complexed forms of its F-ab' fragment. Protein Peptide Lett. 2001; 8:413.

40. Depetris RS, et al. Partial enzymatic deglycosylation preserves the structure of cleaved recombinant HIV-1 envelope glycoprotein trimers. J Biol Chem. 2012; 287:24239. [PubMed: 22645128]

41. Kabsch W. Xds. Acta Crystallogr D Biol Crystallogr. 2010; 66:125. [PubMed: 20124692]

42. McCoy AJ, et al. Phaser crystallographic software. J Appl Crystallogr. 2007; 40:658. [PubMed: 19461840]

43. Winn MD, et al. Overview of the CCP4 suite and current developments. Acta Crystallogr D Biol Crystallogr. 2011; 67:235. [PubMed: 21460441]

44. Adams PD, et al. PHENIX: a comprehensive Python-based system for macromolecular structure solution. Acta Crystallogr D Biol Crystallogr. 2010; 66:213. [PubMed: 20124702]

45. Emsley P, Cowtan K. Coot: model-building tools for molecular graphics. Acta Crystallogr D Biol Crystallogr. 2004; 60:2126. [PubMed: 15572765] 


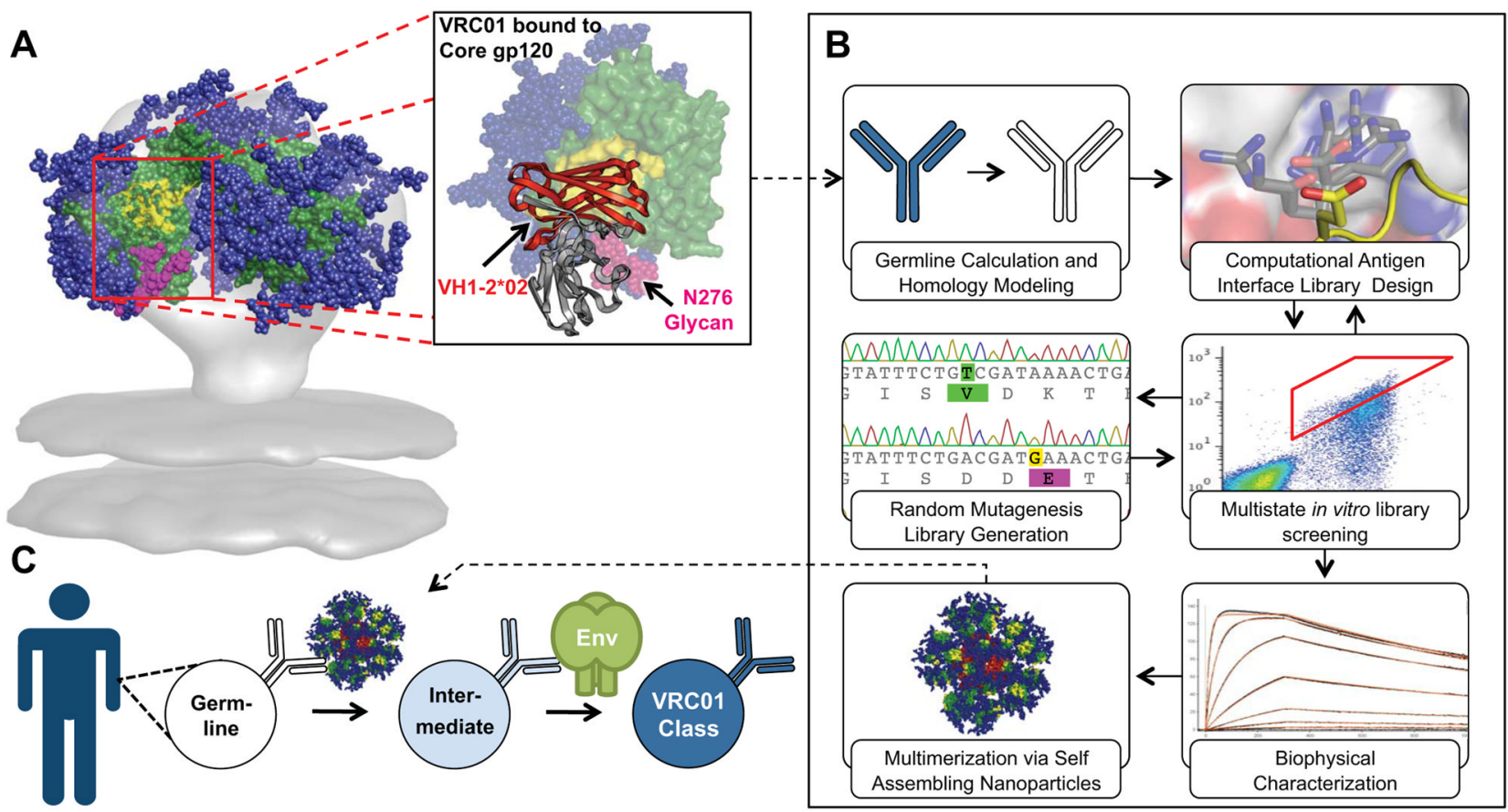

Fig. 1.

Development of a germline (GL)-targeted HIV immunogen.

(A) VRC01-class bNAbs bind to gp120 primarily through paratope residues encoded by VH $1-2 * 02$. Residues on gp120 that interact with CD4 are colored yellow on an Env trimer model and on the surface of core gp120 (green) in complex with VRC01 (PDBID: 3GNB). The region encoded by VH 1-2*02 on VRC01 is colored red and shown as secondary structure rendering. Glycans are shown as blue spheres, except for the critical N276 glycan that is shown in magenta. (B) Engineering of a modified gp120-based nanoparticle capable of activating GL VRC01-class B-cells. (C) This nanoparticle can be used in an HIV-1 vaccine GL-prime-boost strategy to bridge this initial recognition gap and initiate clonal expansion and start somatic hypermutation of VRC01-class bNAbs precursors. 


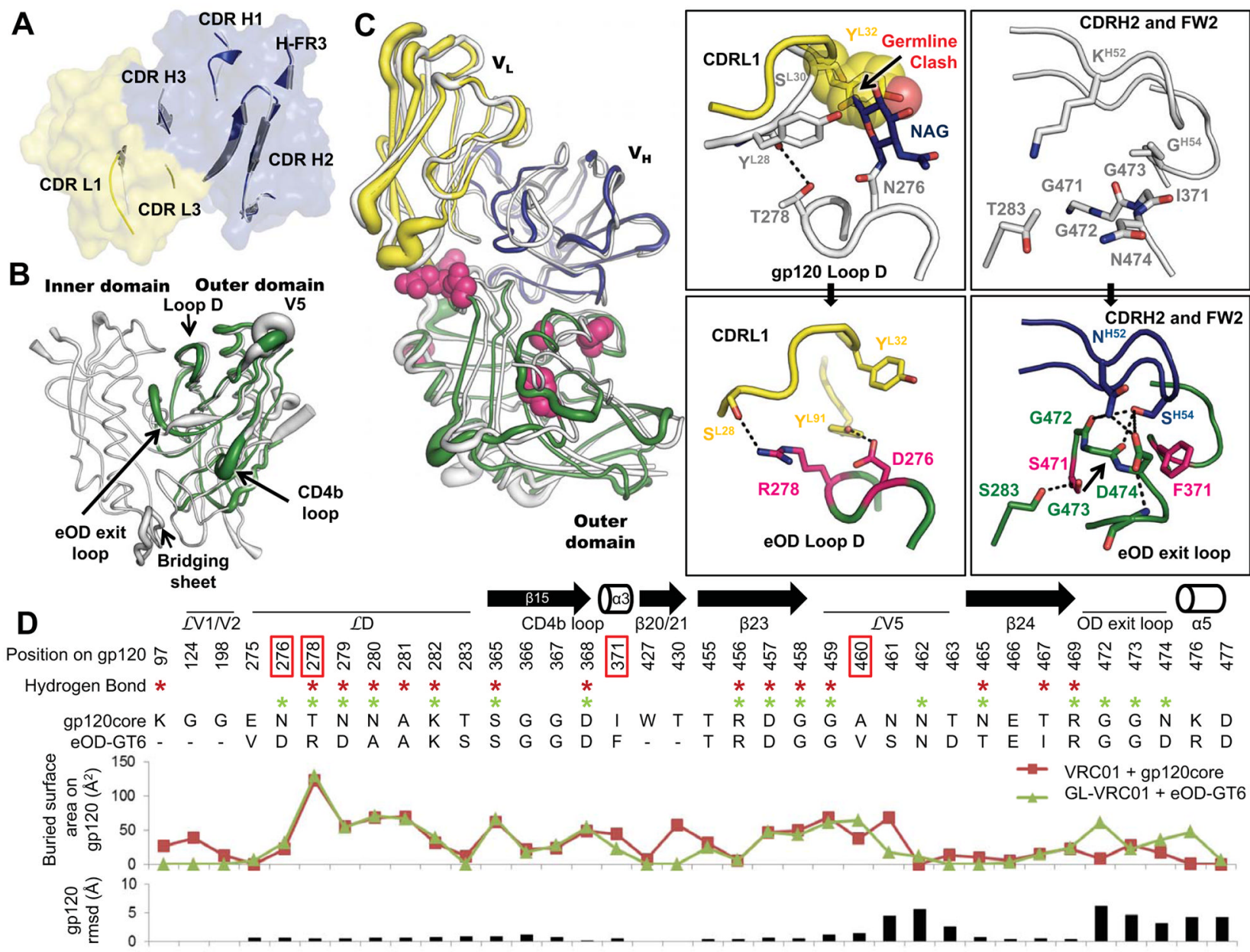

Fig. 2. Structural analysis of GL-VRC01 and eOD-GT6

(A) Comparison between the crystal structure of an unliganded predicted GL-VRC01 antibody (heavy and light chains colored blue and yellow, respectively) and VRC01 bound to gp120 (gray). Variable regions of GL-VRC01 are rendered as a surface. Areas of GLVRC01 and VRC01 that contact gp120, shown as secondary structure cartoons, are similar in conformation. (B) Comparison between the crystal structures of unliganded eOD-GT6 (green) and unliganded gp120 core from HIV-1 strain 93TH057 (PDBID: 3TGT, gray). Structures are rendered according to B-values, with thin and thick lines representing areas possessing low and high flexibility, respectively. (C) Comparison between the crystal structures of GL-VRC01+eOD-GT6 and VRC01+gp120 core. Components are colored as in (A) and (B) and shown as tubes. The positions of the recovered mutations in eOD-GT6 that enable binding of GL-VRC-class Abs are shown as space-fill magenta spheres. The angle of approach of GL-VRC01 and VRC01 to the CD4bs is nearly identical. Key areas where interactions are different between VRC01 on gp120 (upper panels) and GL-VRC01 on eODGT6 (bottom panels) are shown in insets. eOD-GT6 confers germline reactivity by removing a potential clash with the $\mathrm{N} 276$ glycan, as well as by creating additional contacts with loop D (inset I), the OD exit loop (inset II) and V5 (fig. S12). (D) gp120 residues involved in the VRC01+gp120 and GL-VRC01+eOD-GT6 interfaces are compared in sequence, H-bond (stars), surface buried area and RMSD. Interfaces were calculated using PDBePISA (29) and Ca rmsd using Chimera (30). 

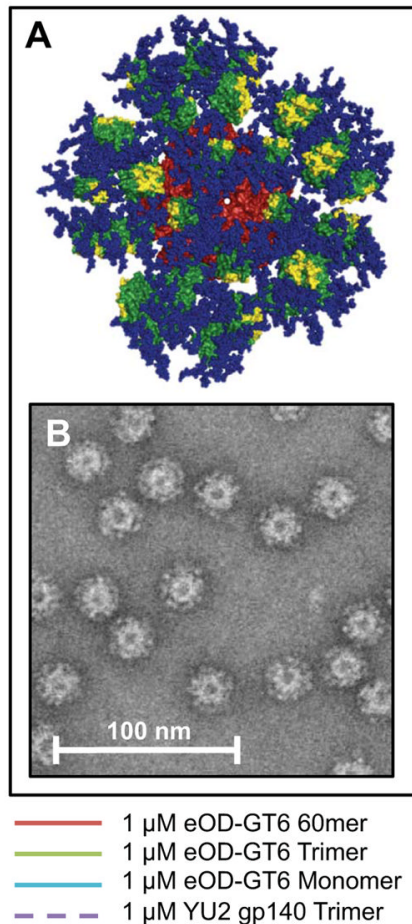

Anti-hCK Activation Control (K46 cell line only)
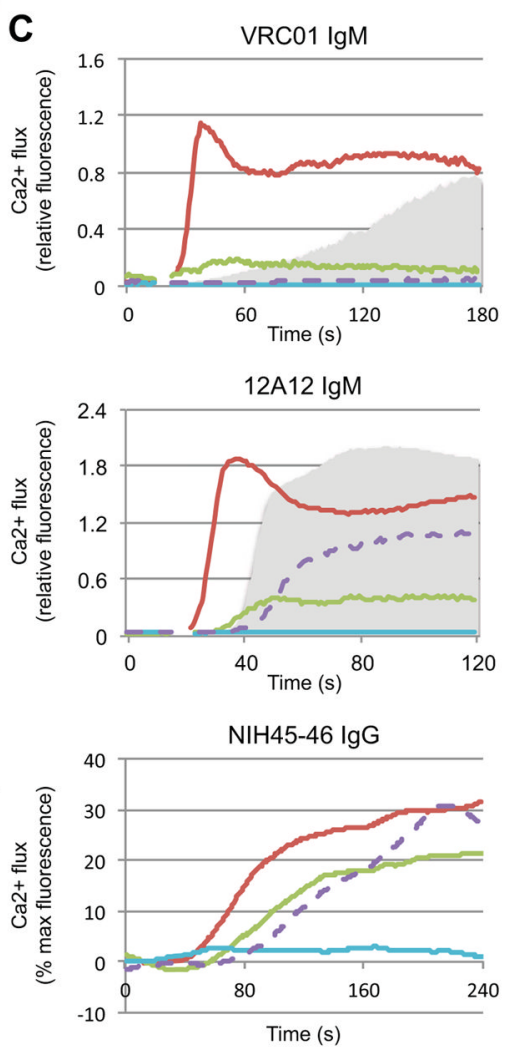
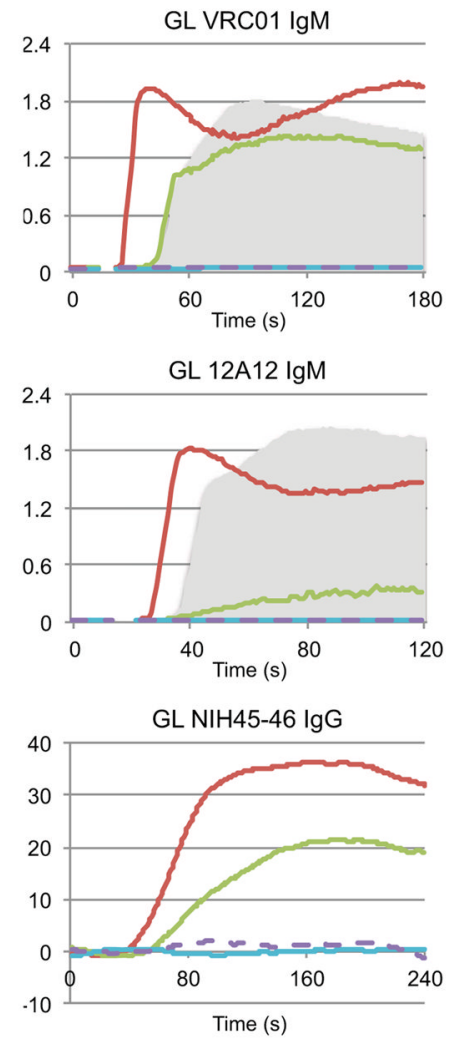

Fig. 3.

A 60-mer eOD-GT6 nanoparticle activates GL and mature VRC01-class B cells. (A) Model representation of the 60-mer eOD-GT6 nanoparticle. eOD-GT6 is colored in green, with residues that interact with $\mathrm{CD} 4$ colored yellow. Glycans are shown as blue spheres and the self-assembling 60mer Lumazine Synthase to which eOD-GT6 is fused is colored red. (B) Raw negative stain electron microscopy images of the 60-mer eOD-GT6 nanoparticle. (C) Calcium flux experiments with various gp120 constructs show that the 60-mer eOD-GT6 nanoparticle activates B cell lines engineered to express either GL or mature VRC01 IgM, 12A12 IgM or NIH45-46 IgG, while a recombinant soluble gp140 trimer activates the B cells expressing mature but not GL VRC01-class Abs. Data for each antibody are representative of at least two separate experiments. 


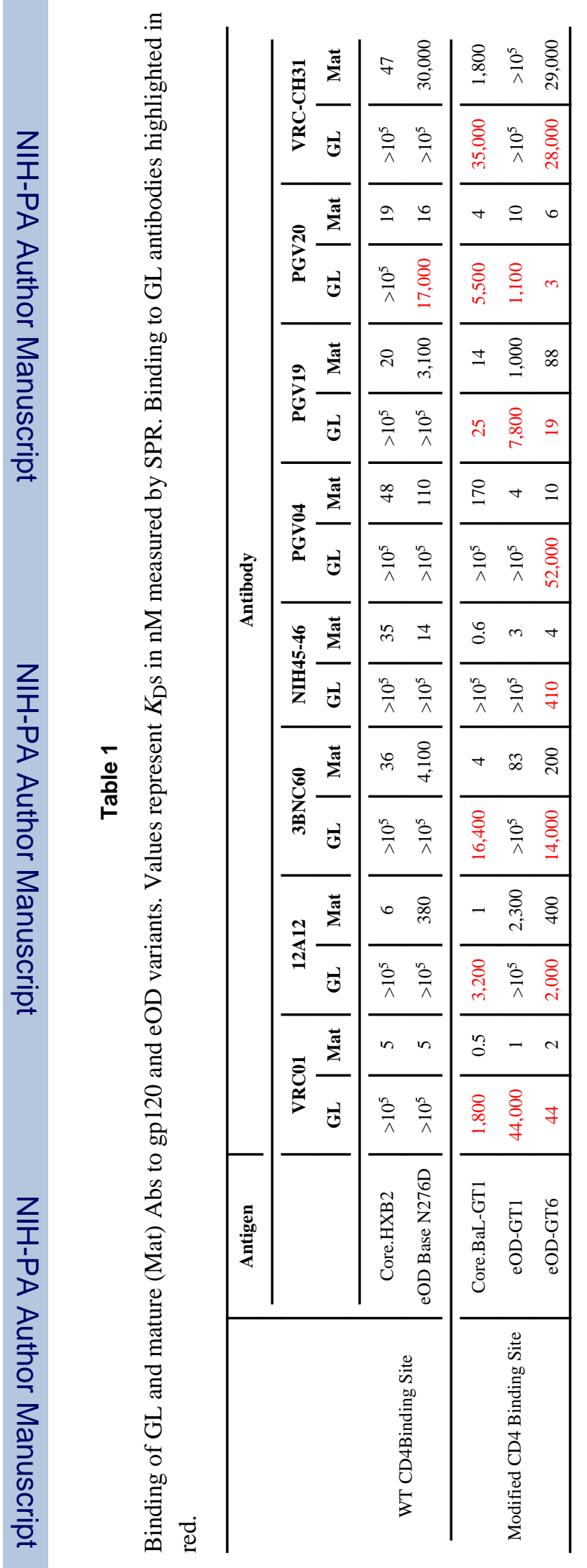


